\title{
Time-optimal Paths for a Dubins airplane
}

\author{
Hamidreza Chitsaz and Steven M. LaValle
}

\begin{abstract}
We consider finding a time-optimal trajectory for an airplane from some starting point and orientation to some final point and orientation. Our model extends the Dubins car [15] to have altitude, which leads to Dubins airplane. We assume that the system has independent bounded control over the altitude velocity as well as the turning rate in the plane. Through the use of the Pontryagin Maximum Principle, we characterize the time-optimal trajectories for the system. They are composed of turns with minimum radius, straight line segments, and pieces of planar elastica. One motivation for determining these elementary pieces is for use as motion primitives in modern planning and control algorithms that consider obstacles.
\end{abstract}

\section{INTRODUCTION}

One can simplify the aircraft control and planning problem, usually in the presence of obstacles, by piecing together, in an appropriate way, a set of elementary trajectories chosen from a library. Such pieces of trajectories that can be combined sequentially to produce more complicated trajectories are called motion primitives [5], [18], [19]. They may even be computed and stored offline, in particular when there are symmetries, to yield speedup in online motion planning applications such as computer games. Finding suitable motion primitives for a robot is an area of recent, active research. One approach is using the optimal trajectories as motion primitives [3].

Many motion planning approaches have relied on good motion primitives, including optimal kinematic trajectories. Latombe successfully used Reeds-Shepp curves in a fast path planner for an indoor mobile robot among obstacles [23]. Conner et al used a set of continuous local feedback control policies and a discrete automaton to plan verifiably correct motions for a mobile robot in a changing environment [14]. Mehta and Egerstedt used optimal control for constructing control programs from a given collection of motion primitives, and also for augmenting the motion primitive set [25]. Frazzoli et al proposed a set of motion primitives, for a six-dimensional aircraft, which contains pieces of optimal trajectories called trim trajectories [18]. Particularly, the optimal trajectories for an aircraft may yield a useful set of motion primitives. We study the time-optimal trajectories for a simplified airplane model in this paper. The time-optimal trajectories play also a crucial role in air traffic management systems [22], [34], [42], e.g. in detecting the safety regions.

H. Chitsaz and S. M. LaValle are with the Department of Computer Science, University of Illinois at Urbana-Champaign, Urbana, IL 61801 \{chitsaz, lavalle\}@cs.uiuc.edu.
Our model is a natural extention of the Dubins car [15], and extends it with an additional configuration variable for the altitude. We consider a simplified kinematic model of the airplane. It always flies forward and the system has independent bounded control over the altitude velocity and the turning rate in the plane. We call such system the Dubins airplane.

The first work on time-optimal paths for a simple airplane model was done by L.E. Dubins [15]. Dubins gave a characterization of time-optimal trajectories for a car with a bounded turning radius. The car always moves forward with constant speed. He used a purely geometrical method to characterize such shortest paths. Later, Reeds and Shepp solved a similar problem in which the car is able to move backward as well [28]. Shortly after Reeds and Shepp, their problem was solved and also refined by Sussmann and Tang [33] and by Boissonnat, Cérézo, and Leblond [7] with the help of optimal control techniques. Souères and Laumond classified the shortest paths for a Reeds-Shepp car into symmetric classes and gave the control synthesis [32]. Balkcom and Mason characterized the time-optimal paths for the differential drive [4], and Chitsaz et al gave a characterization of minimum wheel-rotation paths for the differential drive [11], [12]. In all of those works, the environment is assumed to be unobstructed. The problem becomes more difficult when there are obstacles in the environment. The shortest paths for the Dubins and Reeds-Shepp car and the differential drive among obstacles have also been studied [1], [2], [6], [8], [10], [17], [20], [24], [26], [29], [35], [36], [39].

Walsh, Montgomery, and Sastry used Pontryagin Maximum Principle to plan optimal paths on matrix Lie groups [38]. Specifically, they plan optimal paths for an airplane in $S E(2), S O(3)$, and $S E(3)$. Their cost function is quadratic in the input. In this paper, we consider a different problem in which we minimize time for a system in $S E(2) \times \mathbb{R}$. For algorithms for steering on matrix Lie groups see [30], [37], and for optimal path planning for UAVs with tactical constraints see [40], [41].

The approach that we use to derive optimal trajectories is similar in spirit to the ones used before [4], [7], [12], [13], [31], [33], [38]. The existence of optimal paths in this case follows from Filippov's theorem [9]. We use the Pontryagin Maximum Principle as a necessary condition to rule out nonoptimal paths. We distinguish three cases: low, medium, and high goal altitudes of the airplane. Intuitively, if the goal altitude is low, the airplane has to follow the shortest path for the Dubins car with an unsaturated altitude velocity. If 


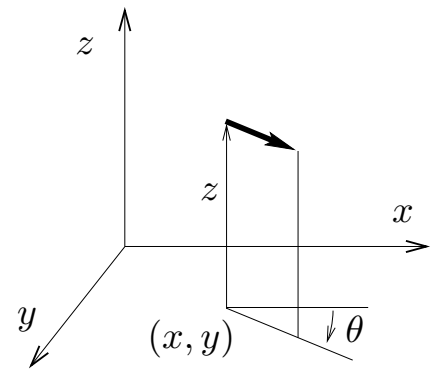

Fig. 1. The Dubins airplane model

the goal altitude is high, the altitude velocity gets saturated and the system has to maneuver until it reaches the goal altitude. For medium altitudes in between low and high, the time-optimal path is either a locally longest curve for the Dubins car or a path composed of turns and pieces of planar elastica [21] with saturated altitude velocity.

Locally longest curves for the Dubins car, which cannot be infinitesimally elongated, play an important role in the airplane time-optimal trajectories for medium altitude. An example of such locally longest curves is a short arc of a circle. Dubins proved that a short arc of a circle is isolated in the space of all bounded curvature plane paths [16]. As a by-product, we characterize locally longest Dubins curves.

\section{Problem Formulation}

The Dubins airplane is a four-dimensional system with its configuration variable denoted by $q=(x, y, z, \theta) \in$ $\mathcal{C}=\mathbb{R}^{3} \times \mathbb{S}^{1}$ in which $x, y$, and $z$ are the coordinates of the airplane in the three-dimensional Euclidean space, and $\theta \in[0,2 \pi)$ is the angle between $x$-axis of the frame and the airplane local longitudinal axis in $x-y$ plane (see Figure 1). Equivalently, the Dubins airplane is the Dubins car, $(x, y, \theta) \in \mathbb{R}^{2} \times \mathbb{S}^{1}$, with an additional configuration variable for altitude, $z$. This model is a simplified model of a real airplane.

The system has independent bounded control of $\dot{\theta}$ and $\dot{z}$. In other words, the system is

$$
\dot{q}=f(q, u)=f_{0}(q)+u_{z} f_{1}(q)+u_{\theta} f_{2}(q)
$$

in which $f_{0}, f_{1}$, and $f_{2}$ are vector fields in the tangent bundle $T C$ of the configuration space. We assume the minimum turning radius and the maximum altitude velocity of the airplane are 1 . In this case, $f_{0}, f_{1}$, and $f_{2}$ are

$$
f_{0}=\left(\begin{array}{c}
\cos \theta \\
\sin \theta \\
0 \\
0
\end{array}\right), f_{1}=\left(\begin{array}{l}
0 \\
0 \\
1 \\
0
\end{array}\right) \text {, and } f_{2}=\left(\begin{array}{l}
0 \\
0 \\
0 \\
1
\end{array}\right) \text {. }
$$

We assume that $\left|u_{z}\right|,\left|u_{\theta}\right| \leq 1$. Thus, the control region is $U=[-1,1]^{2}$ and $\left(u_{z}, u_{\theta}\right) \in U$. The cost functional $J$ to be minimized is time, i.e. $J(u)=\int_{0}^{T} d t$. For every pair of initial and goal configurations, we seek an admissible control, i.e. a measurable function $u:[0, T] \rightarrow U$, that minimizes $J$ while transferring the initial configuration to the goal configuration. Without loss of generality we may assume, throughout this paper, that the initial configuration of the system is $(0,0,0,0) \in \mathcal{C}$. We also denote the goal configuration by $\left(x_{g}, y_{g}, z_{g}, \theta_{g}\right)$. Throughout the paper $\operatorname{sgn}$ is the sign function.

The existence of optimal paths in this case follows from Filippov's theorem [9]. Thus, it is viable to use the Pontryagin Maximum Principle (PMP) for this problem.

\section{Pontryagin Maximum Principle}

Let the Hamiltonian $H: \mathbb{R}^{4} \times \mathcal{C} \times U \rightarrow \mathbb{R}$ be

$$
H(\lambda, q, u)=\langle\lambda, \dot{q}\rangle
$$

in which $\dot{q}$ is given in (1). According to the PMP [27], for every optimal trajectory $q(t)$ defined on $[0, T]$ and associated with control $u(t)$, there exists a constant $\lambda_{0} \geq 0$ and an absolutely continuous vector-valued adjoint function $\lambda(t)=$ $\left(\lambda_{1}(t), \lambda_{2}(t), \lambda_{3}(t), \lambda_{4}(t)\right)$, that is nonzero if $\lambda_{0}=0$, with the following properties along the optimal trajectory:

$$
\begin{aligned}
\dot{\lambda} & =-\frac{\partial H}{\partial q}, \\
H(\lambda(t), q(t), u(t)) & =\max _{z \in U} H(\lambda(t), q(t), z), \\
H(\lambda(t), q(t), u(t)) & \equiv \lambda_{0} .
\end{aligned}
$$

Def 1. An extremal is a trajectory $q(t)$ that satisfies the conditions of the PMP.

In this section, let $q(t)$ be an extremal associated with the adjoint $\lambda(t)$ and the control $u(t)$. Equation (4) can be solved for $\lambda$ to obtain

$$
\lambda(t)=\left(\begin{array}{c}
c_{1} \\
c_{2} \\
c_{3} \\
c_{1} y-c_{2} x+c_{4}
\end{array}\right)
$$

in which $c_{1}, c_{2}, c_{3}$, and $c_{4}$ are constants. Along an extremal, (5) yields the extremal control law

$$
\begin{gathered}
u_{z}=\operatorname{sgn}\left(c_{3}\right) \text { if } c_{3} \neq 0 \\
u_{z} \in[-1,1] \text { if } c_{3}=0 \\
u_{\theta}=\operatorname{sgn}\left(c_{1} y-c_{2} x+c_{4}\right) \text { if } c_{1} y-c_{2} x+c_{4} \neq 0 \\
u_{\theta} \in[-1,1] \text { if } c_{1} y-c_{2} x+c_{4}=0 .
\end{gathered}
$$

If $c_{3}=0$, then (8) implies that $u_{z}$ can have any value within $[-1,1]$. In this case, the following two propositions show that the projection of $q(t)$ onto the $(x, y, \theta)$-space is an extremal for the Dubins car.

Proposition 1. If $c_{3}=0$ and $\lambda_{0} \neq 0$, then the projection of $q(t)$ onto the $(x, y, \theta)$-space is an extremal for the Dubins car, i.e a trajectory of the Dubins car that satisfies the PMP.

Proof. Since $c_{3}=0$ and $H=\lambda_{0} \neq 0$, the vector $\left(\lambda_{1}, \lambda_{2}, \lambda_{4}\right)$ is nonzero. Hence, the projection of $q(t)$ onto 
$(x, y, \theta)$-space satisfies the PMP. Thus, it has to be an extremal for the Dubins car.

Proposition 2. If both $c_{3}=0$ and $\lambda_{0}=0$, then $q(t)$ has zero duration.

Proof. In this case, conditions (6) and (5) imply that $\lambda_{1} \cos \theta+\lambda_{2} \sin \theta+\left|\lambda_{4}\right| \equiv 0$. Thus, the projection of $q(t)$ onto the $(x, y, \theta)$-space is an abnormal extremal for the Dubins car. Abnormal extremals for the Dubins car have zero duration.

If $c_{3} \neq 0$, then the duration of $q(t)$ is $\left|z_{g}\right|$ in which $z_{g}$ is the final altitude, because $u_{z} \equiv 1$ or -1 , depending on the sign of $c_{3}$, by (8). It is possible to have $c_{1}=c_{2}=c_{4}=0$ because $c_{3} \neq 0$. In that case, $u_{\theta}$ can have any value within $[-1,1]$, by (8). This means that the projection of $q(t)$ onto the $(x, y, \theta)$-space can be any feasible path for the Dubins car. However, the length of such path must be $\left|z_{g}\right|$. When does there exist a path of given length for the Dubins car? We will study this question in the following section.

\section{Paths With Given Length for the Dubins CaR}

We desire to plan a path for the Dubins car with prescribed length. Lemma 5.3 in [16] proves that a short arc of circle (of radius 1) is isolated in the space of all admissible paths for the Dubins car. Intuitively, there are no feasible trajectories for the Dubins car between the end points of the arc with a length slightly more than the length of the arc. Whenever there exists a desired path, we pick the one which minimizes a quadratic cost.

Equations of motion for the Dubins car are

$$
\begin{gathered}
\dot{x}=\cos \theta, \\
\dot{y}=\sin \theta, \\
\dot{\theta}=u .
\end{gathered}
$$

Following [38], we pick the path that minimizes $\int_{0}^{T} u^{2} d t$ with given length for this system. If there exists such path, it should satisfy the PMP. Let the Hamiltonian $F: \mathbb{R}^{3} \times\left(\mathbb{R}^{2} \times\right.$ $\left.\mathbb{S}^{1}\right) \times[-1,1] \rightarrow \mathbb{R}$ be

$$
F(\gamma,(x, y, \theta), u)=\langle\gamma,(\cos \theta, \sin \theta, u)\rangle+\gamma_{0} u^{2}
$$

in which $\gamma_{0}$ is a constant. For every desired path $p(t)=$ $(x(t), y(t), \theta(t))$ defined on $[0, T]$ and associated with control $u(t)$, there exists a constant $\gamma_{0} \leq 0$ and an absolutely continuous vector-valued adjoint function $\gamma(t)=$ $\left(\gamma_{1}(t), \gamma_{2}(t), \gamma_{3}(t)\right)$, that is nonzero if $\gamma_{0}=0$, with the following properties along $p(t)$ :

$$
\begin{gathered}
\dot{\gamma}_{1}=-\frac{\partial F}{\partial x}, \\
\dot{\gamma}_{2}=-\frac{\partial F}{\partial y}, \\
\dot{\gamma}_{3}=-\frac{\partial F}{\partial \theta}, \\
F(\gamma(t), p(t), u(t))=\max _{z \in[-1,1]} F(\gamma(t), p(t), z), \\
F(\gamma(t), p(t), u(t)) \equiv K,
\end{gathered}
$$

for some constant $K$. Regular and abnormal extremals, corresponding to $\gamma_{0} \neq 0$ and $\gamma_{0}=0$ respectively, are studied in the following two sections.

\section{A. Regular Extremals}

We may now scale $F$ and assume $\gamma_{0}=-\frac{1}{2}$. Rewriting the Hamiltonian we get $F=\gamma_{1} \cos \theta+\gamma_{2} \sin \theta+\gamma_{3} u-\frac{u^{2}}{2}$. Maximization of $F$ in (19) implies that

$$
u=\left\{\begin{array}{ccc}
-1 & \text { if } & \gamma_{3}<-1 \\
\gamma_{3} & \text { if } & -1 \leq \gamma_{3} \leq 1 \\
1 & \text { if } & \gamma_{3}>1
\end{array}\right.
$$

Equations (16), (17), and (18) can be solved for $\gamma$ to obtain

$$
\gamma(t)=\left(\begin{array}{c}
e_{1} \\
e_{2} \\
e_{1} y-e_{2} x+e_{3}
\end{array}\right)
$$

in which $e_{1}, e_{2}$, and $e_{3}$ are constant. All $e_{i}$ 's cannot be zero, otherwise the extremal is a straight line. Let $\ell: e_{1} y-e_{2} x+$ $e_{3}=0, \ell_{+}: e_{1} y-e_{2} x+e_{3}=1$, and $\ell_{-}: e_{1} y-e_{2} x+e_{3}=-1$ be three lines in the plane. The control law (21) says that $u=\gamma_{3}$ if the car is moving between $\ell_{-}$and $\ell_{+}$. Otherwise, $u=1$ or -1 depending on the position of the car with respect to the lines. Figures 2 and 3 show a few examples of curves that satisfy such control law. These paths are composed of turn with minimum radius, straight line segment, and elastica [21], [38].

\section{B. Abnormal Extremals}

Abnormal extremals correspond to $\gamma_{0}=0$. If $K=0$ in (20), then the extremal is of zero duration. If $K>0$, then the extremal is a time-extremal for the Dubins car. If $K<0$, then the extremal is also an extremal of the functional $I(u)=\int_{0}^{T}-d t$. We call such extremal a locally longest curve, because it can be a local minimum of $I(u)$, or equivalently a local maximum of the length functional.

Further analysis of (19) leads to the following control law:

$$
\begin{gathered}
u=\operatorname{sgn}\left(\gamma_{3}\right) \text { if } \gamma_{3} \neq 0, \\
u \in[-1,1] \text { if } \gamma_{3}=0 .
\end{gathered}
$$

Depending on the sign of $K$, there are two different sets of extremals: time-extremals and locally longest curves.

1) $K>0$, time-extremals: In this case, $F=e_{1} \cos \theta+$ $e_{2} \sin \theta+\left|e_{1} y-e_{2} x+e_{3}\right|=K>0$. Moreover, all $e_{i}$ 's cannot be zero. Thus, the extremal satisfies the PMP with the length cost functional $\int_{0}^{T} d t$. Thus, it is composed of turn with minimum radius and straight line segment. The extremal can tangentially join $\ell$ or diverge from $\ell$. Figure 4 depicts two examples of time-extremals. 
(A)

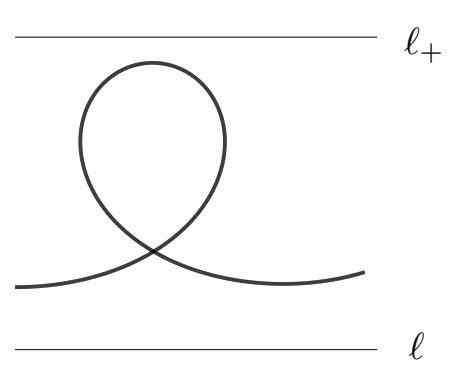

(B)

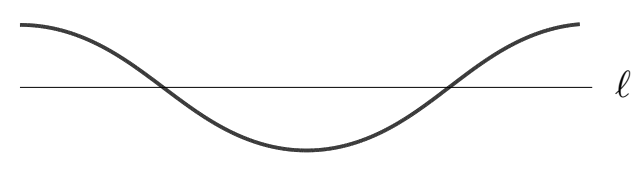

(C)

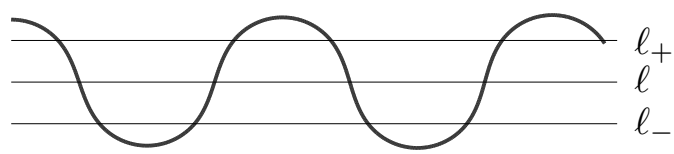

(D)

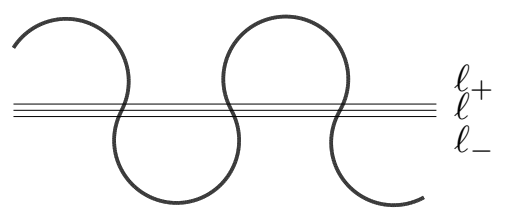

Fig. 2. Some examples of curves with prescribed length for the Dubins car; see also Figure 3

2) $K<0$, locally longest curves: The following constraint holds:

$$
F=e_{1} \cos \theta+e_{2} \sin \theta+\left|e_{1} y-e_{2} x+e_{3}\right|=K<0 .
$$

In this case, the extremal cannot tangentially join $\ell$ unless it violates the constraint. Hence, either $u \equiv 0$ or $u(t)=$ $\operatorname{sgn}\left(e_{1} y(t)-e_{2} x(t)+e_{3}\right)$ and $e_{1} \cos \theta+e_{2} \sin \theta+\mid e_{1} y-$ $e_{2} x+e_{3} \mid<0$. More precisely, either the extremal completely lies on $\ell$, or it is composed of consecutive arcs of circle of length less than $\pi$. In Figure 5, the line $\ell$ and an example of a locally longest curve is shown. Figure 6 shows an elongation
(E)

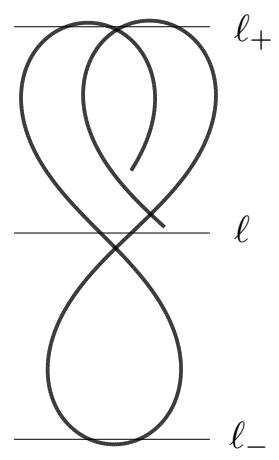

(F)

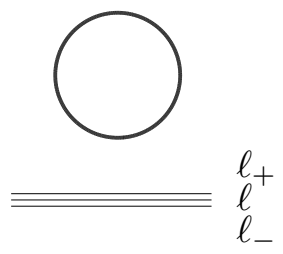

(G)

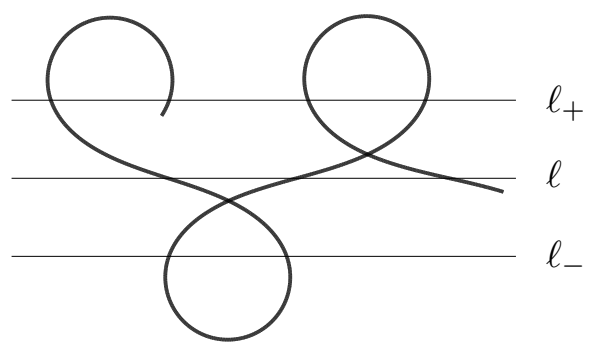

Fig. 3. Continued from Figure 2

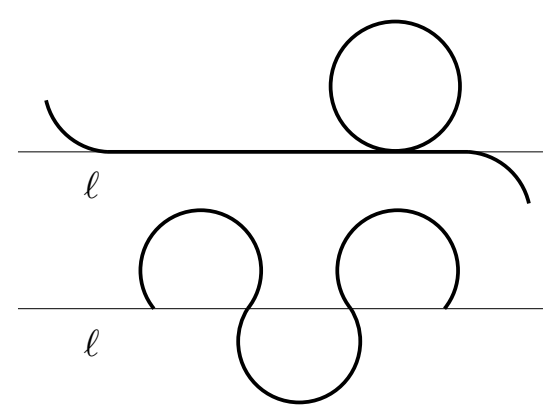

Fig. 4. Two time-extremals

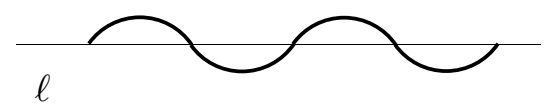

Fig. 5. Locally longest curves for the Dubins car 


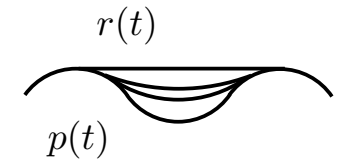

Fig. 6. Elongation of a Dubins shortest path $r(t)$ to a locally longest curve $p(t)$

from $r(t)$, a Dubins shortest path, to a locally longest curve.

\section{Time-optimal Trajectories for the Airplane}

Going back to our original quest, which was to find timeoptimal paths for our airplane, recall that the final altitude plays a major role. We distinguish three cases: low, medium, and high goal altitude. In order to precisely define each case we give the following definition.

Def 2. Let $\Delta$ be the Dubins distance of $\left(x_{g}, y_{g}, \theta_{g}\right)$ from $(0,0,0)$. More precisely, let $\Delta$ denote the duration, or equivalently the length, of the shortest Dubins curve from $(0,0,0)$ to $\left(x_{g}, y_{g}, \theta_{g}\right)$. We call the final altitude low if $\left|z_{g}\right| \leq \Delta$, medium if $\Delta<\left|z_{g}\right|<\Delta+2 \pi$, and high if $\left|z_{g}\right| \geq \Delta+2 \pi$.

\section{A. Time-optimal Trajectories for Low Goal Altitude}

As we mentioned before, following the shortest Dubins curve with an unsaturated altitude velocity is a time-optimal strategy for low goal altitudes. This case corresponds to $c_{3}=0$ in the PMP analysis in Section III. Note that the duration of such trajectory is $\Delta$. It is obvious that there exists no trajectory transferring the system faster from the initial configuration to the goal configuration.

Lemma 1. For a low goal altitude, a time-optimal trajectory for the system (1) consists of the shortest Dubins curve with altitude velocity $u_{z}=\frac{z_{g}}{\Delta}$.

\section{B. Time-optimal Trajectories for High Goal Altitude}

If the goal altitude is high, the system has enough time to follow a helix once it reaches the goal point in the plane and goal orientation. Hence, the shortest Dubins curve followed by a helix all with saturated altitude velocity is a timeoptimal strategy in this case. This case corresponds to $c_{3} \neq 0$ in Section III. The duration of such trajectory is $\left|z_{g}\right|$. There exists no trajectory taking the system faster from the initial to the goal.

Lemma 2. For a high goal altitude, a time-optimal trajectory for the system (1) is composed of two pieces. Along both pieces $u_{z}=\operatorname{sgn}\left(z_{g}\right)$. The projection of the first piece onto the $(x, y, \theta)$-space is the shortest Dubins curve for $\left(x_{g}, y_{g}, \theta_{g}\right)$. The second piece is a helix. The control is $u_{\theta}=\frac{2 \pi}{\left|z_{g}\right|-\Delta}$ along the second piece.

The system first traverses the shortest Dubins curve with saturated altitude velocity along such time-optimal trajectory.
It then traverses a helix, i.e. a full circle in the plane with saturated altitude velocity.

\section{Time-optimal Trajectories for Medium Goal Altitude}

If there is a path for the Dubins car from the initial configuration to the goal configuration in time $\left|z_{g}\right|$, then the time-optimal trajectory for the system corresponds to $c_{3} \neq 0$ in Section III. In this case, the altitude velocity is saturated. If there is no path for the Dubins car from the initial configuration to the goal configuration in time $\left|z_{g}\right|$, then the time-optimal trajectory for the system must correspond to $c_{3}=0$. The altitude velocity is not saturated in this case. Thus, the projection of the time-optimal trajectory onto the $(x, y, \theta)$-space is a Dubins time-extremal. Dubins timeextremals are composed of turn with minimum radius and straight line segments. We presented both cases in Section IV.

\section{CONCLUSIONS}

We introduced the Dubins airplane which extends the Dubins car with altitude. We gave a characterization of the time-optimal trajectories for the Dubins airplane. For low and high final altitudes, the time-optimal trajectories respectively consist of the Dubins curve with unsaturated altitude velocity, and the Dubins curve followed by a helix with saturated altitude velocity. For medium altitudes in between low and high, different cases were recognized. The time-optimal trajectory is either a Dubins extremal (not the shortest) with unsaturated altitude velocity or a Dubins path of certain length with saturated altitude velocity. We gave a method to find a Dubins path with prescribed length if it exists. We also gave an analysis of locally longest curves for the Dubins car, i.e. those paths that may not be infinitesimally elongated. Numerical techniques can be used to compute the control synthesis. Analytical solution for the control synthesis remains open for this problem.

\section{REFERENCES}

[1] P. K. Agarwal, T. Biedl, S. Lazard, S. Robbins, S. Suri, and S. Whitesides. Curvature-constrained shortest paths in a convex polygon. In Proc. ACM Symposium on Computational Geometry, pages 392-401, 1998.

[2] H.-K. Ahn, O. Cheong, J. Matoušek, and A. Vigneron. Reachability by paths of bounded curvature in convex polygons. In Proc. ACM Symposium on Computational Geometry, pages 251-259, 2000.

[3] J. V. Albro and L. E. Bobrow. Optimal motion primitives for a 5 dof experimental hopper. In Proceedings IEEE International Conference on Robotics and Automation, pages 3630- 3635, 2001.

[4] Devin J. Balkcom and Matthew. T. Mason. Time optimal trajectories for bounded velocity differential drive vehicles. Int. J. Robot. Res., 21(3):199-218, March 2002.

[5] C. Belta, A. Bicchi, M. Egerstedt, E. Frazzoli, E. Klavins, and G. J. Pappas. Symbolic planning and control of robot motion: State of the art and grand challenges. IEEE Robotics and Automation Magazine, 14(1):61-70, 2007.

[6] S. Bereg and D. Kirkpatrick. Curvature-bounded traversals of narrow corridors. In Proc. ACM Symposium on Computational Geometry, pages 278-287, 2005.

[7] J.-D. Boissonnat, A. Cérézo, and J. Leblond. Shortest paths of bounded curvature in the plane. J. Intelligent and Robotic Systems, 11:5-20, 1994. 
[8] J. D. Boissonnat and S. Lazard. A polynomial-time algorithm for computing a shortest path of bounded curvature amidst moderate obstacles. In Proc. ACM Symposium on Computational Geometry, pages 242-251, 1996.

[9] Lamberto Cesari. Optimization Theory and Applications: problems with ordinary differetial equations. Springer-Verlag, New York, NY, 1983.

[10] H. Chitsaz and S.M. LaValle. Minimum wheel-rotation paths for differential-drive mobile robots among piecewise smooth obstacles. In IEEE International Conference on Robotics and Automation, 2007.

[11] H. Chitsaz, S.M. LaValle, D.J. Balkcom, and M.T. Mason. An explicit characterization of minimum wheel-rotation paths for differentialdrives. In Proceedings 12th IEEE International Conference on Methods and Models in Automation and Robotics, 2006.

[12] H. Chitsaz, S.M. LaValle, D.J. Balkcom, and M.T. Mason. Minimum wheel-rotation paths for differential-drive mobile robots. In Proceedings IEEE International Conference on Robotics and Automation, 2006.

[13] M. Chyba and S. Sekhavat. Time optimal paths for a mobile robot with one trailer. In IEEE/RSJ Int. Conf. on Intelligent Robots \& Systems, volume 3, pages 1669-1674, 1999.

[14] D. C. Conner, H. Kress-Gazit, H. Choset, A. Rizzi, and G. J. Pappas. Valet parking without a valet. In IEEE/RSJ Int. Conf. on Intelligent Robots \& Systems, 2007

[15] L. E. Dubins. On curves of minimal length with a constraint on average curvature, and with prescribed initial and terminal positions and tangents. American Journal of Mathematics, 79:497-516, 1957.

[16] L. E. Dubins. On plane curves with curvature. Pacific J. Math., 11(2):471481, 1961.

[17] S. Fortune and G. Wilfong. Planning constrained motion. Annals of Mathematics and AI, 3(1):21-82, 1991.

[18] E. Frazzoli, M. A. Dahleh, and E. Feron. Maneuver-based motion planning for nonlinear systems with symmetries. IEEE Trans. on Robotics, 21(6):1077-1091, 2005.

[19] K. Hauser, T. Bretl, K. Harada, and J. C. Latombe. Using motion primitives in probabilistic sample-based planning for humanoid robots. In Workshop on the Algorithmic Foundations of Robotics (WAFR), 2006.

[20] P. Jacobs and J. Canny. Planning smooth paths for mobile robots. In Proceedings IEEE International Conference on Robotics and Automation, pages 2-7, 1989.

[21] V. Jurdjevic. Optimal control problems on Lie groups: Crossroads between geometry and mechanics. In B. Jakubczyk and W. Respondek, editors, Geometry of Feedback and Optimal Control. Marcel-Decker, 1992.

[22] J. Krozel, T. Mueller, and G. Hunter. Free flight conflict detection and resolution analysis. In AIAA Guidance, Navigation and Control Conference AIAA-1996-3763, 1996.

[23] J.-C. Latombe. A fast path planner for a car-like indoor mobile robot. In Proc. Am. Assoc. Artif. Intell., pages 659-665, 1991.

[24] J.-P. Laumond. Finding collision-free smooth trajectories for a nonholonomic mobile robot. In Proc. Int. Joint Conf. on Artif. Intell., pages 1120-1123, 1987.

[25] T. Mehta and M. Egerstedt. An optimal control approach to mode generation in hybrid systems. Nonlinear Analysis: Theory, Methods and Applications, 65(5):963-983, 2006.

[26] P. Moutarlier, B. Mirtich, and J. Canny. Shortest paths for a car-like robot to manifolds in configuration space. Int. J. Robot. Res., 15(1), 1996.

[27] L. S. Pontryagin, V. G. Boltyanskii, R. V. Gamkrelidze, and E. F. Mishchenko. The Mathematical Theory of Optimal Processes. John Wiley, 1962.

[28] J. A. Reeds and L. A. Shepp. Optimal paths for a car that goes both forwards and backwards. Pacific J. Math., 145(2):367-393, 1990.

[29] J. Reif and H. Wang. The complexity of the two dimensional curvature-constrained shortest-path problem. In Proc. Third International Workshop on the Algorithmic Foundations of Robotics, pages 49-57, 1998 .

[30] A. Sarti, G. C. Walsh, and S. Sastry. Steering left-invariant control systems on matrix Lie groups. In IEEE Conf. Decision \& Control, pages $3117-3121,1993$
[31] P. Souères and J.-D. Boissonnat. Optimal trajectories for nonholonomic mobile robots. In J.-P. Laumond, editor, Robot Motion Planning and Control, pages 93-170. Springer, 1998.

[32] P. Souères and J. P. Laumond. Shortest paths synthesis for a car-like robot. In IEEE Transactions on Automatic Control, pages 672-688, 1996.

[33] Héctor Sussmann and Guoqing Tang. Shortest paths for the ReedsShepp car: A worked out example of the use of geometric techniques in nonlinear optimal control. Technical Report SYNCON 91-10, Dept. of Mathematics, Rutgers University, 1991.

[34] C. Tomlin, G. J. Pappas, and S. Sastry. Conflict resolution for air traffic management: A study in multiagent hybrid systems. IEEE Trans. on Automatic Control, 43(4), 1998.

[35] M. Vendittelli, J.P. Laumond, and C. Nissoux. Obstacle distance for car-like robots. IEEE Transactions on Robotics and Automation, 15(4):678-691, 1999.

[36] M. Vendittelli, J.P. Laumond, and P. Souères. Shortest paths to obstacles for a polygonal car-like robot. In IEEE Conf. Decision \& Control, 1999.

[37] G. Walsh, A. Sarti, and S. Shankar Sastry. Algorithms for steering on the group of rotations. Technical Report UCB/ERL M93/44, EECS Department, University of California, Berkeley, 1993.

[38] G. C. Walsh, R. Montgomery, and S. Sastry. Optimal path planning on matrix Lie groups. In IEEE Conf. Decision \& Control, volume 2 , pages 1258-1263, 1994.

[39] H. Wang and P. K. Agarwal. Approximation algorithms for curvatureconstrained shortest paths. In Proc. Annual ACM-SIAM Symp. on Discrete algorithms, pages 409-418, 1996.

[40] H. Wong, V. Kapila, and R. Vaidyanathan. UAV optimal path planning using c-c-c class paths for target touring. In IEEE Conf. Decision \& Control, pages 1105-1110, 2004.

[41] G. Yang and V. Kapila. Optimal path planning for unmanned air vehicles with kinematic and tactical constraints. In IEEE Conf. Decision \& Control, pages 1301-1306, 2002.

[42] Y. Zhao and R. L. Schultz. Deterministic resolution of two aircraft conflict in free flight. In AIAA Guidance, Navigation, and Control Conference AIAA-1997-3547, 1997. 